\title{
Offline Data Processing Software for the JUNO Experiment
}

Xingtao Huang*, Teng Li

School of Physics, Shandong University, Jinan 250100, China

E-mail: huangxt@sdu.edu.cn

Jiaheng Zou, Tao Lin, Weidong Li, Ziyan Deng, Guofu Cao

Institute of High Energy Physics, Beijing 100049, China

On behalf of the JUNO Collaboration

JUNO is a multi-purpose neutrino experiment designed to determine the neutrino mass hierarchy and precisely measure oscillation parameters. The offline data processing of JUNO is based on the newly designed and developed SNiPER framework which provides flexible event management buffer, efficient event execution controlling, user-friendly interfaces and so on. The event data model of JUNO is based on the ROOT TObject. A new type of smart pointer, SmartRef, is designed to meet the special multi-event correlation requirements of neutrino experiments. SmartRef uses the Universally Unique Identifier to handle the references of event data objects, both in memory and in ROOT files. The Input/Output system supports the lazy-loading of event objects when reading data from ROOT files. So far, JUNO offline data processing software has been successfully used for the optimization of detector performance as well as study of reconstruction and physics performance.

38th International Conference on High Energy Physics

3-10 August 2016

Chicago, USA

${ }^{*}$ Speaker. 


\section{Introduction}

The JUNO (Jiangmen Underground Neutrino Observatory) is a multi-purpose neutrino experiment proposed to determine neutrino mass hierarchy and precisely measure oscillation parameters [1]. Now it is under preparation in southern China. Anti-electron neutrinos are detected via the inverse beta decay (IBD) reaction which yields correlated prompt and delayed signals resulting in separate detector triggers. Natural radioactive decays and cosmic muon induced processes yield background signals that potentially mix with or mimic the IBD signals. The JUNO Offline software is designed to facilitate the convenient correlated analysis of prompt and delayed signals from candidate IBD events while maintaining associations between the various processing stages. In addition, the software meets general data processing requirements.

\section{Offline Software System}

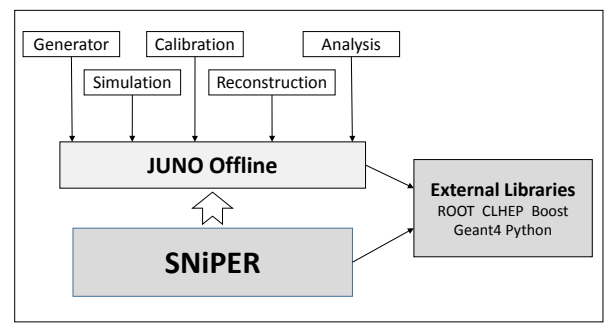

Figure 1: Overview of offline data processing software of JUNO

The JUNO Offline software system is based on the SNiPER (Software for Non-collider Physics ExpeRiment) framework [2]. JUNO Offline is composed of SNiPER plugin components, as shown in Fig. 1, with dependencies on external packages including Geant4, CLHEP, ROOT and Boost.

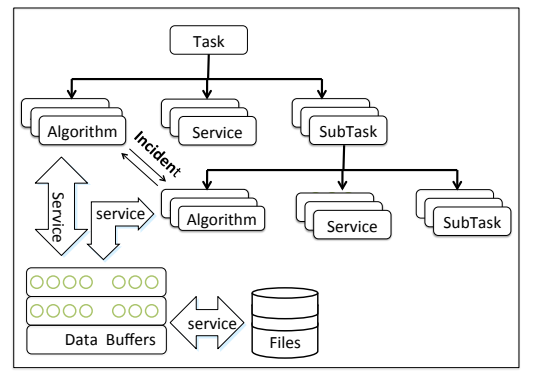

Figure 2: Main components of SNiPER

JUNO Offline includes all software specific to the JUNO experiment, including event data model, detector geometry description, input/output system, simulation, reconstruction and physics analysis tools. Main components of SNiPER are shown in Fig. 2.

- Algorithm is the basic unit for data processing and provides the interface for users and developers to write their own codes in order to be dynamically loaded by SNiPER. Simulation, calibration and reconstruction are all implemented as Algorithms. 
- DataBuffer is a dynamically allocated region of memory that holds multiple events in order to facilitate correlation analysis. The size is configured with a time window. In each execution, an event acts as the anchor of the time window and its adjacent events in the time window are cached in the DataBuffer simultaneously. During the event loops, the anchor event moves forward one by one, and events in the time window are accordingly updated.

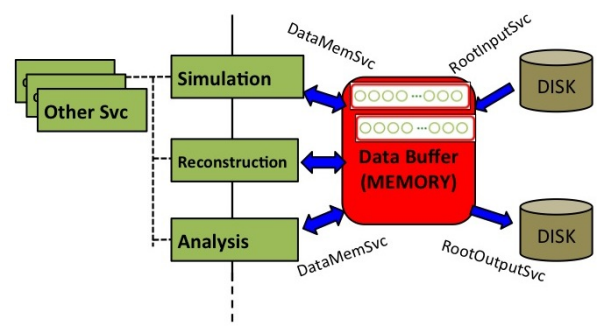

Figure 3: Management and Access of Event Objects

- Services provide data movement between the DataBuffer and Algorithms and provide input/output between the DataBuffer and ROOT files, as shown in Fig. 3.

- Tasks act as lightweight application managers, as shown in Fig. 4, containing algorithms, DataBuffer, services and other subtasks. Tasks are dynamically configurable and orchestrate the sequential execution of the contained algorithms. Different tasks are executed with an incident mechanism, enabling event mixing and splitting to be implemented.

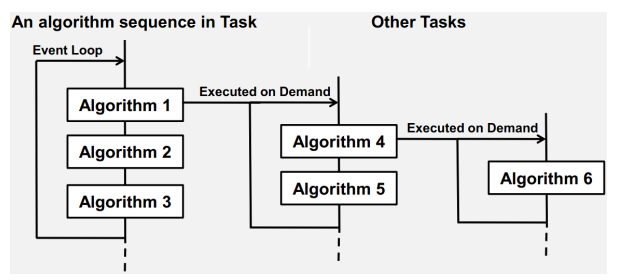

Figure 4: Task and Execution Controlling

- Incidents are named software triggers that are fired by some components and that are caught by other components to take corresponding actions. In Fig. 4, incidents are used to implement executions from one task to the other task.

- Event Data Model, as shown in Fig. 5, uses separate header and event object pairs for each data processing stage. All objects use the ROOT TObject base class. The lightweight header objects contain only characteristic information or tags while the event objects contain the full information. Header tags are used to enable fast event selection.

- Neutrino detector data production and simulation studies depend on correlations between objects across multiple processing stages to a much greater degree than collider experiments. In order to support this requirement, a new type of SmartRef [3] pointer has been designed and implemented based on Universally Unique Identifiers (UUID) allowing flexible referencing 


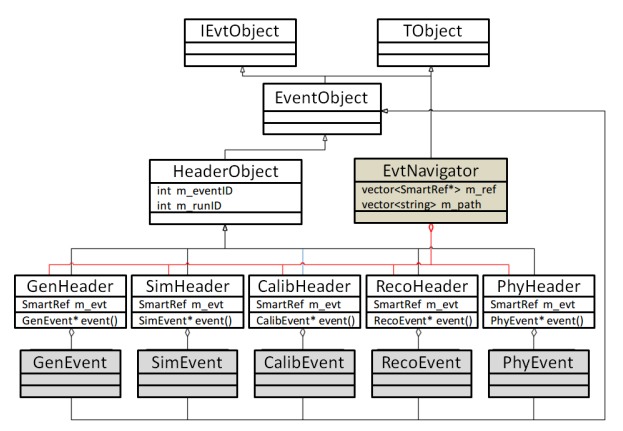

Figure 5: Scheme of JUNO Event Data Model

and lazy loading across multiple event data objects and processing stages both in memory and in ROOT files. As illustrated in Fig. 5, correlations of event objects at different stages are implemented with Navigators which hold a set of SmartRefs to objects in different stages.

\section{Status and Outlook}

The Offline data processing software for the JUNO experiment has been designed and implemented based on the SNiPER framework which was created to meet the offline processing requirements of neutrino experiments. Extensive correlation requirements have been met using a DataBuffer approach with a new SmartRef pointer and Navigator system operating across events and processing stages with lazy loading from ROOT files.

\section{Acknowledgements}

This work is supported by the Joint Large-Scale Scientific Facility Funds of the NSFC and CAS (U1532258), the Program for New Century Excellent Talents in University (NCET-13-0342) and the Shandong Natural Science Funds for Distinguished Young Scholar (JQ201402).

\section{References}

[1] Fengpeng An et al. Neutrino physics with JUNO, J.Phys.G43 (2016) 3030401

[2] J. H. Zou et al, J. Phys. Conf. Ser., 664(7): 072053 (2015)

[3] T. Li , X.T. Huang, J. Phys. Conf. Ser., 762(1): 012001 (2016) 